\title{
Origin of the counterintuitive dynamic charge in the transition metal dichalcogenides
}

\author{
Nicholas A. Pike, ${ }^{1}$ Benoit Van Troeye, ${ }^{2}$ Antoine Dewandre, ${ }^{1}$ Guido Petretto, ${ }^{2}$ Xavier Gonze, ${ }^{2}$ \\ Gian-Marco Rignanese, ${ }^{2}$ and Matthieu J. Verstraete ${ }^{1}$ \\ ${ }^{1}$ Nanomat/Q-Mat/CESAM, Université de Liège and European Theoretical Spectroscopy Facility, B-4000 Liège, Belgium \\ ${ }^{2}$ Université Catholique de Louvain, Institute of Condensed Matter and Nanosciences (IMCN) \\ and European Theoretical Spectroscopy Facility, B-1348 Louvain-la-Neuve, Belgium
}

(Received 10 February 2017; published 18 May 2017)

\begin{abstract}
Despite numerous studies of transition metal dichalcogenides, the diversity of their chemical bonding characteristics and charge transfer is not well understood. Based on density functional theory we investigate their static and dynamic charges. The dynamic charge of the transition metal dichalcogenides with trigonal symmetry are anomalously large, while in their hexagonally symmetric counterparts, we even observe a counterintuitive sign, i.e., the transition metal takes a negative charge, opposite to its static charge. This phenomenon, so far never remarked on or analyzed, is understood by investigating the perturbative response of the system and by investigating the hybridization of the molecular orbitals near the Fermi level. Furthermore, a link is established between the sign of the Born effective charge and the process of $\pi$ backbonding from organic chemistry. Experiments are proposed to verify the calculated sign of the dynamical charge in these materials. Employing a high-throughput search we also identify other materials that present counterintuitive dynamic charges.
\end{abstract}

DOI: 10.1103/PhysRevB.95.201106

\section{INTRODUCTION}

The expanding interest in the transition metal dichalcogenides (TMDs) stems from their tunability and a wide variety of potential applications, ranging from batteries to electronic devices [1-3]. Indeed, by adjusting either their chemical composition or the number of deposited layers, one can tune their electronic, vibrational, and magnetic properties in a remarkable manner that cannot be imitated in other twodimensional or layered materials. In particular, the TMDs offer high carrier mobilities [4-6] and a strain-dependent indirect to direct band-gap transition [7] that are both crucial for future electronic and electro-optic applications [8,9]. Still, while the electronic properties of these materials are now relatively well known [10], the character of their chemical bonds is, interestingly, quite diverse, and, to the best of our knowledge, not yet fully understood. For example, while $\mathrm{ZrS}_{2}$ is reported as extremely ionic [11,12], $\mathrm{MoS}_{2}$ and $\mathrm{WS}_{2}$ are reported to possess both ionic and covalent characteristics [13,14], and $\mathrm{TiS}_{2}$ was reported both metallic and semiconducting, in experiment and in theory [15-17].

In this Rapid Communication, a deeper understanding of the bonding characteristics and charge transfer in the TMDs is given due to density functional theory (DFT) [18] and density functional perturbation theory (DFPT) [19-21] calculations. One common way to characterize the charge distribution within this theory is based on the partition of the electronic density of the system into constituent atoms, following, e.g., Bader [22] or Hirshfeld [23]. While conceptually simple, this notion of "static" charge is, unfortunately, ambiguous [24,25]. Contrarily, the Born effective charge (BEC) [21], arising from the change of the dipole moment due to an atomic perturbation, is uniquely defined as it corresponds to the dynamic polarization response due to an atomic displacement. It governs, for example, the splitting between the transverse optical (TO) and longitudinal optical (LO) vibrational modes [21]. It has been argued [24] that all the various operational charge definitions (including static and dynamic charges) share a single principal component, which seems an intuitive result. However, in various materials, e.g., ferroelectric perovskites, the BEC is observed to be anomalously large with respect to its static nominal value, albeit without a change in sign [25-27].

In this Rapid Communication, the critical differences between the dynamic BEC and static Bader charge are highlighted for the hexagonal TMDs (h-TMDs). Indeed, the BEC calculations indicate that the transition metal atom takes the negative charge, while the static charges lead to the opposite conclusion, be they nominal, Bader or Hirshfeld. This is a clear occurrence of such a counterintuitive discrepancy between static and dynamic charge signs. The calculated BEC sign and magnitude agree with recent DFPT calculations in Refs. [28,29], but disagree in sign with Ref. [30]. The h-TMDs contrast strongly with the trigonal TMDs (t-TMDs), where the sign of the static charge and the BEC agree. In what follows, our first-principles calculations of the static and dynamic charge are outlined and then, in Sec. III, an analysis of these charges helps to determine the origin of the sign change and an investigation of the molecular orbital diagrams leads to a connection with $\pi$ backbonding. Finally, in Sec. IV a discussion of this phenomenon and concluding remarks are given. Additional calculation details and results are provided in the Supplemental Material (SM) [31].

\section{NUMERICAL METHODS AND RESULTS}

Our calculations are performed using the ABINIT software package [32-34] with the GGA-PBE exchange-correlation functional [18,35-38], corrected by Grimme's DFT-D3 functional for the dispersion corrections due to long-range electronelectron correlations $[39,40]$. With the inclusion of this van der Waals functional, our calculations reproduce the in-plane and out-of-plane experimental lattice parameters to within $0.7 \%$ [41-46]. Details on the norm-conserving pseudopotentials $[47,48]$, the convergence parameters (plane-wave expansion cutoff energy and Brillouin-zone sampling), and the structural parameters are found in the SM [31]. 
(a)

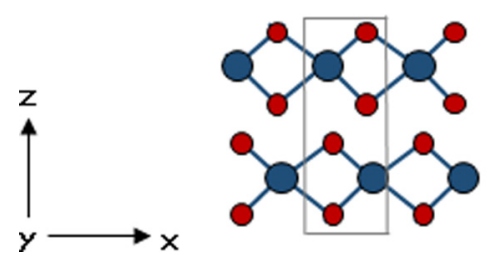

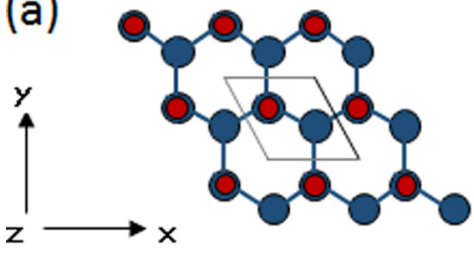

(b)
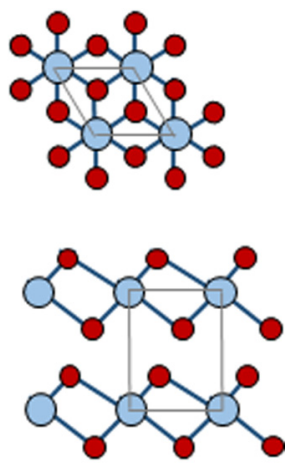

FIG. 1. A sketch of the bulk structure of (a) $\mathrm{MoS}_{2}$ and (b) $\mathrm{TiS}_{2}$ with the transition metals in dark blue (Mo) and light blue (Ti) and the chalcogen, $\mathrm{S}$, in red. The h-TMDs have AB stacking along the $z$ axis and the t-TMDs have AA stacking along the $z$ axis. The light-gray box indicates the unit cell of each compound.

We investigate the Bader charge, $Z^{B}$, and BEC, $Z^{*}$, for the bulk $M X_{2} \mathrm{~h}$-TMDs, where $M=\mathrm{Mo}, \mathrm{W}$, and $X=\mathrm{S}$, Se, Te, as well as for $\mathrm{TiS}_{2}$ and $\mathrm{TiSe}_{2}$, two semiconducting $\mathrm{t}$-TMDs. There are noticeable differences in the symmetries and stacking of each type of compound, as shown in Fig. 1 where the top and side views of the bulk h- and t-TMDs are shown. The calculation of the BEC uses DFPT with the charge neutrality condition imposed. All calculations of the static and dynamic charges use the relaxed geometries for the individual compounds.

Table I reports the calculated BECs and Bader charges for the previously introduced TMDs, alongside experimental data extracted from infrared spectra [12,49-52]. Infrared spectroscopy only provides a measure of the magnitude of the BEC. Thus, the calculated BECs must be compared accordingly. For both $\mathrm{h}$ - and t-TMDs, the absolute value of computed BECs compare relatively well with the available experimental data. However, the BECs are anomalously large in t-TMDs, as they

TABLE I. Computed BEC, absolute value of the experimental BEC, static Bader charge, and BPDC for the transition metal atom, $M$, in the h- and t-TMDs. The BPDC is defined in the main text. The BECs and Bader charges are given for the two principal directions $x x$ and $z z$, with all other elements either zero or found by symmetry. The chalcogen charges are exactly $-1 / 2$ the corresponding transition metal charges.

\begin{tabular}{|c|c|c|c|c|c|c|}
\hline & \multicolumn{4}{|c|}{ Born effective charge $[e]$} & \multirow[b]{2}{*}{ Bader $[e]$} & \multirow[b]{2}{*}{$\mathrm{BPDC}[e]$} \\
\hline & \multirow[b]{2}{*}{$Z_{M, x x}^{*}$} & \multirow[b]{2}{*}{$Z_{M, z z}^{*}$} & \multicolumn{2}{|c|}{ Expt. [12,49-52] } & & \\
\hline & & & $\left|Z_{M, x x}^{*}\right|$ & $\left|Z_{M, z z}^{*}\right|$ & $Z_{M, z}^{B}$ & $Z_{M, z}^{B, *}$ \\
\hline $\mathrm{MoS}_{2}$ & -1.090 & -0.628 & 1.1 & 0.4 & 1.155 & 0.635 \\
\hline $\mathrm{MoSe}_{2}$ & -1.906 & -0.955 & 2.1 & 0.5 & 0.910 & 0.652 \\
\hline $\mathrm{MoTe}_{2}$ & -3.095 & -1.544 & 3.4 & & 0.575 & 0.752 \\
\hline $\mathrm{WS}_{2}$ & -0.491 & -0.426 & 0.4 & 0.2 & 1.400 & \\
\hline $\mathrm{WSe}_{2}$ & -1.243 & -0.776 & 1.7 & 0.5 & 1.081 & \\
\hline $\mathrm{TiS}_{2}$ & 6.323 & 1.208 & 6.0 & 2.2 & 1.764 & 1.330 \\
\hline $\mathrm{TiSe}_{2}$ & 6.122 & 0.855 & 9.2 & 2.1 & 1.599 & \\
\hline
\end{tabular}

differ strongly from both their corresponding nominal (chalcogen ion -2 , transition metal ion +4 , with signs in agreement with Pauling electronegativity scale) and static charges [25], while in the h-TMDs, the dynamical charges are observed to be counterintuitive, with the transition metal and chalcogen atoms taking the negative and positive charges, respectively, in disagreement with the corresponding nominal and static charges. These counterintuitive BECs for h-TMDs were also reported recently in monolayer TMDs [28,29], but the authors did not pay attention to or explain the calculated sign. This is a case in which the sign of the static charge and BEC disagree clearly, with the absolute difference being more than three electronic charges in the extreme case of $\mathrm{MoTe}_{2}$, in contrast with the early belief [24] that all the various operational charge definitions share a single principal component.

\section{CHARGE AND MOLECULAR ORBITAL ANALYSIS}

The comparison between static and dynamical charges is quite delicate as they represent different physical quantities: the static charge corresponds to a partition of the ground-state electronic density, while the dynamic charge corresponds directly to the dynamic response due to an atomic perturbation. Still, one can construct a dynamical charge based on the static charge by taking into account the change of Bader charge with an atomic displacement [25] computed by finite differences. This newly constructed Bader partitioned dynamic charge (BPDC), denoted $Z^{B, *}$, includes additional effects, i.e., the charge (de)localization.

In plane, the displacement of an atom generates a global electron current, whose contribution to the BPDC cannot be determined by a simple finite difference approach. On the contrary, as the interactions between the layers are negligible, an atomic displacement in the out-of-plane direction leads only to a weak intralayer charge transfer. For simplicity, the atomic displacements in the out-of-plane direction are assumed to correspond to no electron current due to the large distance between the layers. The corresponding charges are reported in Table I. While this dynamic correction to the static Bader charge is negative in most cases, in agreement with the sign of $\Delta Z$, it is clearly too small to fully explain the sign of the BECs in h-TMDs. Thus, the counterintuitive charges can be explained in terms of a local change in polarization around the atoms that cannot be quantified by a partitioning approach [25]. This is confirmed by the analysis of the perturbed density with respect to an electric field perturbation, presented in Fig. 2, which is localized close to the Mo atoms. Changes within this region cannot be quantified by the Bader approach, in contrast to $\mathrm{TiS}_{2}$ where most contributions come from outside the $\mathrm{Ti}$ Bader volume as shown in the SM [31].

Consequently, a more direct analysis of the BECs is crucial for our understanding of the discrepancies between the dynamical charges of the $\mathrm{h}$ - and t-TMDs represented by $\mathrm{MoS}_{2}$ and $\mathrm{TiS}_{2}$. The band-by-band decomposition [54] and localization tensor [55] are unable to bring any simple or conclusive explanations on the difference of BECs between these two materials as shown in Table S2 of the SM [31]. Thus, it is necessary to analyze the different contributions to the BECs which are given explicitly, for example, in Ref. [21]. Neglecting the separable part, the dynamic screening 
(a) $\mathrm{MoS}_{2}$ : Mo Plane

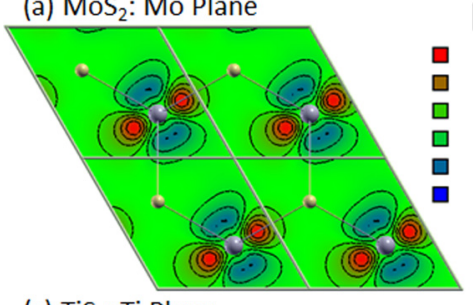

(c) $\mathrm{TiS}_{2}$ : Ti Plane

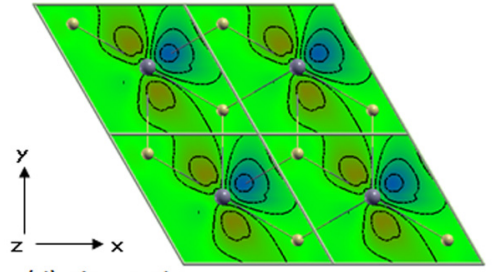

(d) $\mathrm{TiS}_{2}$ : S Plane

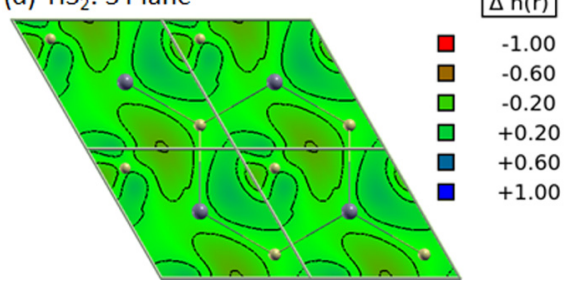

FIG. 2. Contour plots and isosurfaces of the change in the electronic density, in units of $e / \mathrm{bohr}^{-2}$ integrated along the $z$ axis, due to an electric field perturbation along the $x$ axis for $\mathrm{MoS}_{2}$ and $\mathrm{TiS}_{2} . \mathrm{MoS}_{2}$ : (a) the in-plane change in the electronic density around a Mo atom and (b) the induced change in the electronic density for a single formula unit of $\mathrm{MoS}_{2}$. The change in the electronic density around $\mathrm{S}$ is negligible (not presented). $\mathrm{TiS}_{2}$ : (c) the in-plane change in electronic density around $\mathrm{Ti}$ and in (d) around $\mathrm{S}$ and (e) a plot of the induced change in the electronic density for $\mathrm{TiS}_{2}$. The atomic colors indicate the transition metal atom, in gray, and the chalcogen atom, in tan. Visualization provided by XCRYSDEN [53].

component, given in Eq. (S3) of the SM [31], depends on, first, the change in the electronic wave function due to an electric field perturbation and, second, on the change of electronic potential due to an atomic displacement. While the change in potential does not vary qualitatively between $\mathrm{MoS}_{2}$ and $\mathrm{TiS}_{2}$ (see Fig. S2 of the SM [31]), their first-order density responses differ significantly, as illustrated in Fig. 2. In $\mathrm{MoS}_{2}$, this change of electronic density with an external electric field is localized around the Mo atom [Fig. 2(b)] and takes a hybridized $d$-orbital shape, while in $\mathrm{TiS}_{2}$ it is delocalized along the Ti-S bond [Fig. 2(e)].

This localization/delocalization of the electronic density responses in $\mathrm{MoS}_{2}$ and $\mathrm{TiS}_{2}$, which leads to the opposite character of the BEC between these materials, should arise from differences in orbital hybridization, orbital symmetry, and/or electronic configuration of h-TMDs and t-TMDs. With this in mind, and in order to understand the differences in hybridization between $\mathrm{MoS}_{2}$ and $\mathrm{TiS}_{2}$, the molecular orbital (MO) diagrams [56] are presented, based on the work of Stiefel et al. [57]. Similar to the latter work, the molecular orbitals of $\mathrm{MoS}_{2}$ and $\mathrm{TiS}_{2}$ monolayers are labeled using the irreducible representation of the molecular orbitals for a single formula unit within these compounds. Therefore, the point-group symmetries $D_{3 h}$ and $D_{3 d}$ are used for $\mathrm{MoS}_{2}$ and

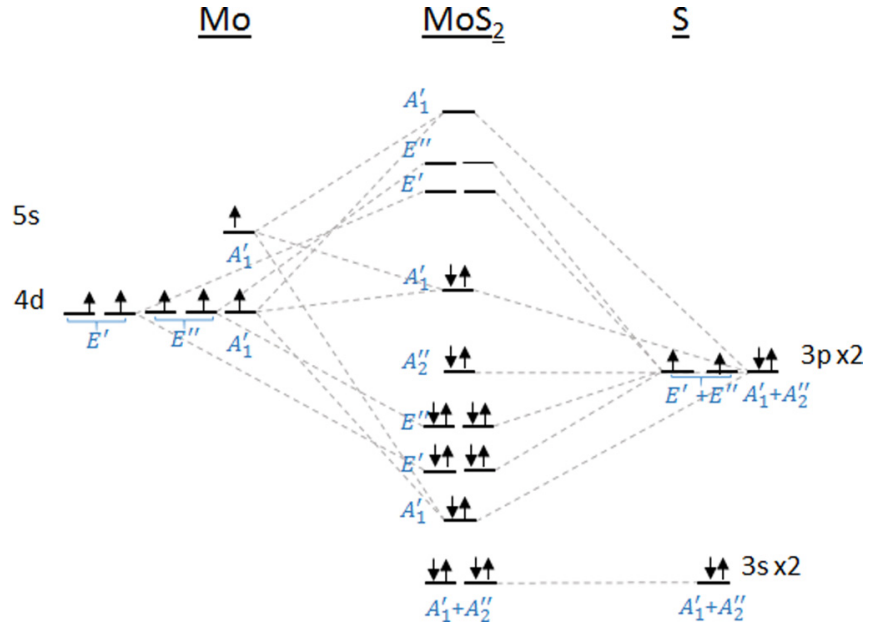

FIG. 3. Molecular orbital diagram of $\mathrm{MoS}_{2}$. The symmetry notation of the point group $D_{3 h}$, in blue, labels the symmetry type of the molecular orbital, and the light-gray dashed lines are the symbolic links between the atomic and molecular orbitals.

$\mathrm{TiS}_{2}$, respectively. The orbital energy ordering was obtained by a direct comparison to the projected band analysis of $\mathrm{MoS}_{2}$ and $\mathrm{TiS}_{2}$ presented in the SM [31].

The MO diagram of $\mathrm{MoS}_{2}$ is presented in Fig. 3. It indicates that the lowest $A_{1}^{\prime}$, as well as the lowest $E^{\prime}$ and $E^{\prime \prime}$ molecular orbitals of $\mathrm{MoS}_{2}$, are all bonding orbitals. According to the projected orbital analysis, this $A_{1}^{\prime}$ orbital contains mostly $\mathrm{S}$ orbital contributions, while the $E^{\prime}$ and $E^{\prime \prime}$ orbitals are located on both Mo and $\mathrm{S}$ atoms. The $A_{2}^{\prime \prime}$ orbital, arising from the interaction between $p_{z}$ orbitals of $\mathrm{S}$, does not hybridize with the Mo atomic orbitals. The last occupied orbital-also an $A_{1}^{\prime}$ - lies closest to the Fermi energy, and is an antibonding orbital arising from Mo, with a small amount of S. The first unoccupied states correspond to antibonding $E^{\prime}$ and $E^{\prime \prime}$ states containing both Mo and $\mathrm{S}$ atoms.

The MO diagram of $\mathrm{TiS}_{2}$ is depicted in Fig. 4 where the valence bands of $\mathrm{TiS}_{2}$ contain a majority of atomiclike $S$ states, in agreement with the projected band analysis in the SM [31],

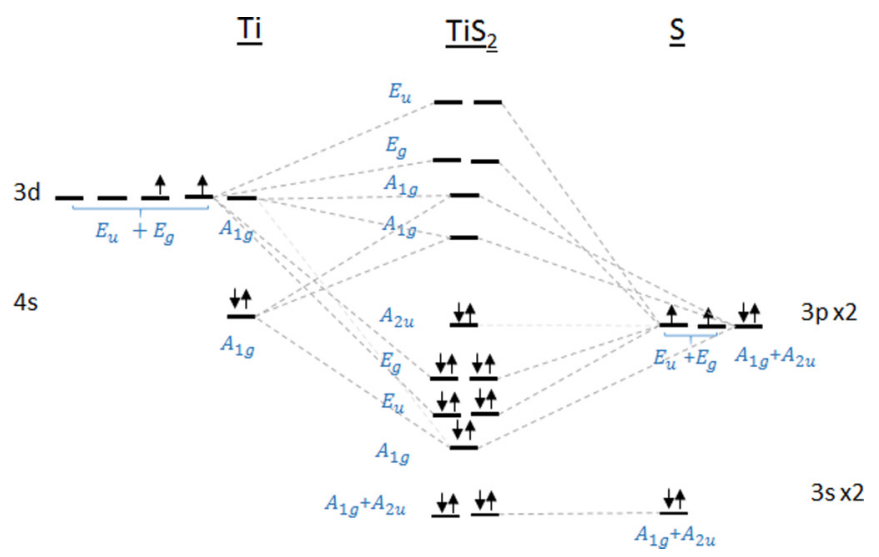

FIG. 4. Molecular-orbital diagram of $\mathrm{TiS}_{2}$. The symmetry notation of the point group $D_{3 d}$, in blue, labels the symmetry type of the molecular orbital, and the light-gray dashed lines are the symbolic links between the atomic and molecular orbitals. 
indicating charge transfer from $\mathrm{Ti}$ to $\mathrm{S}$. Note the inversion of the atomic Ti $4 s$ and $3 d$ states, compared to $\mathrm{MoS}_{2}$, and the bonding character of the highest occupied MO.

In the case of the h-TMDs, a parallel can be made with the $\pi$-backbonding effect in organic chemistry, a process in which not only a $\sigma$ bond forms between a metal atom and a ligand but also an additional $\pi$ bond that involves an antibonding state of the ligand [58]. This second bond transfers charge back to a $d$ state of the metal atom, leading to a weakening of the ligand internal bond. In the case of $\mathrm{MoS}_{2}$, the Mo atoms share their electrons with the $\mathrm{S}$ atoms, which transfer back their electrons to the Mo atoms (antibonding state composed of $p_{z}$ orbitals) in order to fill the $4 d_{z^{2}}$ orbital of Mo. The presence of two types of atomic orbitals from the transition metal, $s$ and $d$, with different spatial extents, is critical in both the wellknown $\pi$-backbonding phenomena and the counterintuitive and anomalous BECs.

With these MO diagrams in mind, and focusing on the out-of-plane direction, the origin of the sign of the BEC can be understood in the h-TMDs. Similar to the monolayer, the highest occupied molecular-orbital level of bulk h-TMDs (split due to $\mathrm{AB}$ stacking) remains antibonding. This orbital is rather localized around Mo due to its antibonding character, in contrast to the other bonds in this compound, which are found to be mostly delocalized (with Mo and S characteristics). However, this localized bond, corresponding to a superposition of Mo $4 d_{z^{2}}$, Mo $5 s$, and S $3 p$ states is especially sensitive to atomic displacements. This bond gives rise to the local change in polarization that was described previously in this Rapid Communication and to the counterintuitive sign of the BECs in the h-TMDs. On the contrary, the last occupied orbitals of $\mathrm{TiS}_{2}$ are all bonding and delocalized, and thus do not lead to any local change of polarization. This explanation remains valid for monolayer h-TMDs, and for the in-plane components of the BECs as well. The symmetry and hybridization determine the sequence of MO levels, and the number of transition metal valence electrons determines whether an antibonding orbital is filled, with the consequent anomalous BEC sign.

\section{DISCUSSION AND CONCLUSIONS}

Experimentally it is difficult to determine the sign of the BECs, as most relevant quantities, in particular the LO-TO splitting, depend on the BEC squared, and not its sign. However, the BEC is a physical observable (the polarization when atoms are displaced), and its sign should be measurable in a system where the mirror plane symmetry is broken: a movement of the metal ion will lead to an asymmetric effect as a function of applied field or strain. There exist other layered TMDs similar to the h-TMDs, but with lower lattice symmetry, in particular $\mathrm{TcS}_{2}, \mathrm{ReS}_{2}$, and $\mathrm{ReSe}_{2}$ which belong to triclinic space groups. Their unit cells are more complex, with inequivalent metal sites and buckled chalcogens, but we also find they present anomalous BEC signs. In these cases, the Raman susceptibility tensor can be used to determine the sign of the BECs: the components of the tensor are linearly dependent on the BEC [59]. The sign can be deduced by angle-resolved Raman as in Wolverson et al. [60], which shows clearly that $\mathrm{ReSe}_{2}$ has anomalous BEC signs (details in the SM [31]). Finally, it may also be possible to measure the sign of the BEC using $\mathrm{x}$-ray absorption spectroscopy in a strong electric field, which is atom specific [61].

In order to understand the origins of this phenomenon, we have started a high-throughput analysis combining publicly available data [62,63] and our own BEC DFPT calculations. After a first screening of more than 200 compounds we have identified a set of additional cases with BECs which are counterintuitive with respect to static charges. These range over different elements and symmetries, proving that counterintuitive BEC are more widespread than might be expected. Among these compounds, some chalcogenides such as $\mathrm{RuSe}_{2}$ and $\mathrm{FeS}_{2}$ share with $\mathrm{MoS}_{2}$ the key feature of having antibonding states at the top of the valence bands, even if they appear in cubic and orthorhombic phases, respectively. This shows that the explanation detailed here applies to a more general class of materials than just the h-TMDs.

In conclusion, the counterintuitive sign of the Born effective charges in the hexagonal TMDs comes from an important local change of polarization around the transition metal atom, caused by an occupied antibonding orbital close to the Fermi level involving the $d$ electrons of the transition metal and the $p$ electrons of the chalcogens. A parallel is made with the $\pi$-backbonding effect in organic chemistry, and methods are proposed to confirm the sign of the computed BECs experimentally. Additionally, counterintuitive BECs have been identified in several other compounds.

\section{ACKNOWLEDGMENTS}

The authors gratefully acknowledge discussions with $\mathrm{Ph}$. Ghosez and funding by the Belgian Fonds National de la Recherche Scientifique FNRS under Grants No. PDR T.1077.15-1/7 (N.A.P and M.J.V.) and No. PDR T.1031.14 (G.P. and G.-M.R.) and for a FRIA Grant (B.V.T.). M.J.V and A.D. acknowledge support from ULg and from the Communauté Française de Belgique (ARC AIMED 15/19-09). Computational resources have been provided by the Université Catholique de Louvain (CISM/UCL); the Consortium des Equipements de Calcul Intensif en Fédération Wallonie Bruxelles (CECI), funded by FRS-FNRS G.A. 2.5020.11; the Tier-1 supercomputer of the Fédération Wallonie-Bruxelles, funded by the Walloon Region under G.A. 1117545; and by PRACE-3IP DECI grants, on ARCHER and Salomon (ThermoSpin, ACEID, OPTOGEN, and INTERPHON 3IP G.A. FP7 RI-312793 and 13 G. A. 653838 of H2020).

N.A.P. and B.V.T. contributed equally to this work.
[1] M. Pumera, Z. Sofer, and A. Ambrosi, Layered transition metal dichalcogenides for electrochemical energy generation and storage, J. Mater. Chem. A 2, 8981 (2014).
[2] D. Jariwala, V. K. Sangwan, L. J. Lauhon, T. J. Marks, and M. C. Hersam, Emerging device applications for semiconducting two-dimensional transition metal dichalcogenides, ACS Nano 8, 1102 (2014). 
[3] A. A. Tedstone, D. J. Lewis, and P. O'Brian, Synthesis, properties, and applications of transition metal-doped layered transition metal dichalcogenides, Chem. Mater. 28, 1965 (2016).

[4] A. Gupta, T. Sakthivel, and S. Seal, Recent developments in 2D materials beyond graphene, Prog. Mater. Sci. 73, 44 (2015).

[5] G. R. Bhimanapati, Z. Lin, V. Meunier, Y. Jung, J. Cha, S. Das, D. Xiao, Y. Son, M. S. Strano, V. R. Cooper et al., Recent advances in two-dimensional materials beyond graphene, ACS Nano 9, 11509 (2015).

[6] Q. H. Wang, K. Kalantar-Zadeh, A. Kis, J. N. Coleman, and M. S. Strano, Electronic and optoelectronics of two-dimensional transition metal dichalcogenides, Nat. Nanotechnol. 7, 699 (2012).

[7] C. Espejo, T. Rangel, A. H. Romero, X. Gonze, and G.-M. Rignanese, Band structure tunability in $\mathrm{MoS}_{2}$ under interlayer compression: A DFT and $G W$ study, Phys. Rev. B 87, 245114 (2013).

[8] B. Radisavljevic, A. Radenovic, J. Brivio, V. Giacometti, and A. Kis, Single-layer $\mathrm{MoS}_{2}$ Transistors, Nat. Nanotechnol. 6, 147 (2011).

[9] N. Kumar, S. Najmaei, Q. Cui, F. Ceballos, P. M. Ajayan, J. Lou, and H. Zhao, Second harmonic microscopy of monolayer $\mathrm{MoS}_{2}$, Phys. Rev. B 87, 161403(R) (2013).

[10] A. Kuc, T. Heine, and A. Kis, Electronic properties of transitionmetal dichalcogenides, MRS Bull. 40, 577 (2015).

[11] R. M. White and G. Lucovsky, Chemical bonding and structure in layered transition metal dichalcogenides, Solid State Commun. 11, 1369 (1972).

[12] H. P. Vaterlaus and F. Levy, Phonons and free carriers in group IVB transition-metal dichalcogenides, J. Phys. C: Solid State Phys. 18, 2351 (1985).

[13] G. Lucovsky, R. M. White, J. A. Benda, and J. F. Revelli, Infrared-reflectance spectra of layered group-IV and group-VI transition-metal dichalcogenides, Phys. Rev. B 7, 3859 (1973).

[14] J.-Q. Li and D.-P. Huang, The energy band structures and chemical bonds of solid state compounds with low-dimensional structures Part I: Delocalized $d-p \pi$ bonding and conductivity of transition metal compounds with low-dimensional structures, J. Mol. Struct.: THEOCHEM 363, 23 (1996).

[15] C. M. Fang, R. A. de Groot, and C. Haas, Bulk and surface electronic structure of $1 T-\mathrm{TiS}_{2}$ and $1 T-\mathrm{TiSe}_{2}$, Phys. Rev. B 56, 4455 (1997).

[16] Y.-H. Liu, S. H. Porter, and J. E. Goldberger, Dimensional reduction of a layered metal chalcogenide into a $1 \mathrm{D}$ near-IR direct band gap semiconductor, J. Am. Chem. Soc. 134, 5044 (2012).

[17] S. Sharma, T. Nautiyal, G. S. Singh, S. Auluck, P. Blaha, and C. Ambrosh-Draxl, Electronic structure of $1 T-\mathrm{TiS}_{2}$, Phys. Rev. B 59, 14833 (1999).

[18] R. M. Martin, Electronic structure: Basic Theory and Practice Methods (Cambridge University Press, Cambridge, UK, 2004).

[19] S. Baroni, S. de Gironcoli, A. D. Corso, and P. Gainnozzi, Phonons and related crystal properties from density-functional perturbation theory, Rev. Mod. Phys. 73, 515 (2001).

[20] X. Gonze, First-principles responses of solids to atomic displacements and homogeneous electric fields: Implementation of a conjugate-gradient algorithm, Phys. Rev. B 55, 10337 (1997).

[21] X. Gonze and C. Lee, Dynamical matrices, Born effective charges, dielectric permittivity tensors, and, interatomic force constants from density-functional perturbation theory, Phys. Rev. B 55, 10355 (1997).

[22] R. F. W. Bader, Atoms in molecules, Acc. Chem. Res. 18, 9 (1985).

[23] F. L. Hirshfeld, Bonded-atom fragments for describing molecular charge densities, Theor. Chim. Acta 44, 129 (1977).

[24] J. Meister and W. H. E. Schwarz, Principal components of ionicity, J. Phys. Chem. 98, 8245 (1994).

[25] P. Ghosez, J.-P. Michenaud, and X. Gonze, Dynamical atomic charges: The case of $\mathrm{ABO}_{3}$ compounds, Phys. Rev. B 58, 6224 (1998).

[26] P. Ghosez, X. Gonze, P. Lambin, and J.-P. Michenaud, Born effective charges of barium titanate: Band-by-band decomposition and sensitivity to structural features, Phys. Rev. B 51, 6765 (1995).

[27] F. Detraux, P. Ghosez, and X. Gonze, Anomalously large Born effective charges in cubic $\mathrm{WO}_{3}$, Phys. Rev. B 56, 983 (1997).

[28] T. Sohier, M. Calandra, and F. Mauri, Two-dimensional Fröhlich interaction in transition-metal dichalcogenide monolayers: Theoretical modeling and first-principles calculations, Phys. Rev. B 94, 085415 (2016).

[29] M. Danovich, I. L. Aleiner, N. D. Drummond, and V. I. Fal'ko, Fast relaxation of photo-excited carriers in 2D transition metal dichalcogenides, IEEE J. Sel. Top. Quantum Electron. 23, 6000105 (2017).

[30] C. Ataca, H. Sahin, and S. Ciraci, Stable, single-layer MX2 transition-metal oxides and dichalcogenides in a honeycomblike structure, J. Phys. Chem. C 116, 8983 (2012).

[31] See Supplemental Material at http://link.aps.org/supplemental/ 10.1103/PhysRevB.95.201106 for additional information on the calculation methods, dynamic partition of the Bader charge, band-by-band decomposition, and possible experimental measurements which includes Refs. [9,20,25,33,35,36,38, $40-48,53,55,59,60]$.

[32] X. Gonze, G.-M. Rignanese, M. Verstraete, J.-M. Beuken, Y. Pouillon, R. Caracas, F. Jollet, M. Torrent, G. Zerah, M. Mikami, $\mathrm{Ph}$. Ghosez, M. Veithen, J.-Y. Raty, V. Olevano, F. Bruneval, L. Reining, R. Godby, G. Onida, D. R. Hamann, and D. C. Allan, A brief introduction to the ABINIT software package, Zeit. Kristallogr. 220, 558 (2005).

[33] X. Gonze, B. Amadon, P. M. Anglade, J.-M. Beuken, F. Bottin, P. Boulanger, F. Bruneval, D. Caliste, R. Caracas, M. Cote, T. Deutsch, L. Genovese, P. Ghosez, M. Giantomassi, S. Goedecker, D. Hamann, P. Hermet, F. Jollet, G. Jomard, S. Leroux et al., ABINIT: First-principles approach to material and nanosystem properties, Comput. Phys. Commun. 180, 2582 (2009).

[34] X. Gonze, F. Jollet, F. A. Araujo, D. Adams, B. Amadon, T. Applencourt, C. Audouze, J.-M. Beuken, J. Bieder, A. Bokhanchuk et al., Recent developments in the ABINIT software package, Comput. Phys. Commun. 205, 106 (2016).

[35] J. P. Perdew, K. Burke, and M. Ernzerhof, Generalized Gradient Approximation Made Simple, Phys. Rev. Lett. 77, 3865 (1996).

[36] M. Fuchs and M. Scheffler, Ab initio pseudopotentials for electronic structure calculations of poly-atomic systems using density-functional theory, Comput. Phys. Commun. 119, 67 (1999).

[37] M. Torrent, F. Jollet, F. Bottin, G. Zérah, and X. Gonze, Implementation of the projector augmented-wave method in the 
ABINIT code: Application to the study of iron under pressure, Comput. Mater. Sci. 42, 337 (2008).

[38] M. A. L. Marques, M. J. T. Oliveira, and T. Burnus, Libxc: A library of exchange and correlation functionals for density functional theory, Comput. Phys. Commun. 183, 2272 (2012).

[39] B. V. Troeye, M. Torrent, and X. Gonze, Interatomic force constants including the DFT-D dispersion contribution, Phys. Rev. B 93, 144304 (2016).

[40] S. Grimme, S. Ehrlich, and L. Goerigk, A consistent and accurate $a b$ initio parametrization of density functional dispersion correction (DFT-D) for the 94 elements H-Pu, J. Chem. Phys. 132, 154104 (2010).

[41] R. Dickinson and L. Pauling, The crystal structure of molybdinite, J. Am. Chem. Soc. 45, 1466 (1923).

[42] L. H. Brixner, Preparation and properties of the single crystalline $\mathrm{AB}_{2}$-type selenides and tellurides of niobium, tantalum, molybdenum, and tungsten, J. Inorg. Nucl. Chem. 24, 257 (1962).

[43] A. Berkdemir, H. R. Guiterrez, A. R. Botello-Mendez, N. PereaLopez, A. L. Elias, C-I. Chia, V. H. Crespi, F. Lopez-Urias, J-C. Charlier, H. Terrones, and M. Terrones, Identification of individiual and few layers of $\mathrm{WS}_{2}$ using Raman spectroscopy, Sci. Rep. 3, 1755 (2013).

[44] M. Traving, M. Boehme, L. Kipp, M. Skibowski, F. Starrost, E. E. Krosovskii, A. Perlov, and W. Schattke, Electronic structure of $\mathrm{WSe}_{2}$ : A combined photoemission and inverse photoemission study, Phys. Rev. B 55, 10392 (1997).

[45] C. Wan, Y. Wang, N. Wang, and K. Koumoto, Low-thermalconductivity $(M S)_{1+x}\left(\mathrm{TiS}_{2}\right)_{2}(M=\mathrm{Pb}, \mathrm{Bi}, \mathrm{Sn})$ misfit layer compounds for bulk thermoelectrics, Materials 3, 2606 (2010).

[46] P. Chen, Y-H. Chan, X-Y. Fang, Y. Zhang, M. Y. Chou, S.-K. Mo, Z. Hussain, A. V. Fedorov, and T. C. Chaing, Charge density wave transition in single-layer titanium diselenide, Nat. Commun. 6, 8943 (2015).

[47] P. E. Blochl, Projected augmented-wave method, Phys. Rev. B 50, 17953 (1994).

[48] F. Jollet, M. Torrent, and N. Holzwarth, Generation of projected augmented-wave atomic data: A 71 element validated table in XML format, Comput. Phys. Commun. 185, 1246 (2014).

[49] Q.-C. Sun, X. S. Xu, L. I. Vergara, R. Rosentsveig, and J. L. Musfeldt, Dynamical charge and structural strain in inorganic fullerenelike $\mathrm{MoS}_{2}$ nanoparticles, Phys. Rev. B 79, 205405 (2009).

[50] T. J. Wieting, A. Grisel, and F. Levy, Interlayer bonding and localized charge in $\mathrm{MoSe}_{2}$ and $\alpha-\mathrm{MoTe}_{2}$, Physica B 99, 337 (1980).
[51] R. D. Luttrell, S. Brown, J. Cao, J. L. Musfeldt, R. Rosentsveig, and R. Tenne, Dynamics of bulk versus nanoscale $\mathrm{WS}_{2}$ : Local strain and charging effects, Phys. Rev. B 73, 035410 (2006).

[52] S.-I. Uchida and S. Tanaka, Optical phonon modes and localized effective charges of transition-metal dichalogenides, J. Phys. Soc. Jpn. 45, 153 (1978).

[53] A. Kokalj, Computer graphics and graphical user interface as tools in simulations of matter at the atomic scale, Comput. Mater. Sci. 28, 155 (2003).

[54] P. Ghosez and X. Gonze, Band-by-band decompositions of the Born effective charges, J. Phys.: Condens. Matter 12, 9179 (2000).

[55] M. Veithen, X. Gonze, and P. Ghosez, Electron localization: Band-by-band decomposition and application to oxides, Phys. Rev. B 66, 235113 (2002).

[56] P. D. Fleischauer, J. R. Lince, P. A. Bertrand, and R. Bauer, Electronic structure and lubrication properties of $\mathrm{MoS}_{2}$ : A qualitative molecular orbital approach, Langmuir 5, 1009 (1989).

[57] E. I. Stiefel, R. Eisenberg, R. C. Rosenberg, and H. B. Gray, Characterization and electronic structure of six-coordinate trigonal-prismatic complexes, J. Am. Chem. Soc. 88, 2956 (1966).

[58] G. L. Meissler, P. J. Fischer, and D. A. Tarr, Inorganic Chemistry, 5th ed. (Pearson, New York, 2014).

[59] M. Veithen, X. Gonze, and P. Ghosez, Nonlinear optical susceptibilities, Raman efficiencies, and electro-optic tensors from first-principles density functional perturbation theory, Phys. Rev. B 71, 125107 (2005).

[60] D. Wolverson, S. Crampin, A. S. Kazemi, A. Ilie, and S. J. Bending, Raman spectra of monolayer, few-layer and bulk $\mathrm{ReSe}_{2}$ : An anisotropic layered semiconductor, ACS Nano 8, 11154 (2014).

[61] V. Ney, W. K. Ollefs, A. Rogalev, and A. Ney, X-ray absorption spectroscopy in electrical fields: An element-selective probe of atomic polarization, Phys. Rev. B 93, 035136 (2016).

[62] A. Jain, S. P. Ong, G. Hautier, W. Chen, W. D. Richards, S. Dacek, S. Cholia, D. Gunter, D. Skinner, G. Ceder, and K. A. Persson, The Materials Project: A materials genome approach to accelerating materials innovation, APL Mater. 1, 011002 (2013).

[63] S. Curtarolo, W. Setyawan, S. Wang, J. Xue, K. Yang, R. H. Taylor, G. L. W. Hart, S. Sanvito, M. Buongiorno-Nardelli, N. Mingo, and O. Levy, AFLOWLIB.ORG: A distributed materials properties repository from high-throughput $a b$ initio calculations, Comput. Mater. Sci. 58, 227 (2012). 